\title{
Hierarchical Learning Machines and Neuroscience of Visual Cortex
}

\author{
Tomaso Poggio \\ Center for Biological \& Computational Learning \\ McGovern Institute \\ Computer Science and Artificial Intelligence Laboratory \\ Department of Brain and Cognitive Sciences \\ Massachusetts Institute of Technology \\ tp@csail.mit.edu
}

Learning is the gateway to understanding intelligence and to reproducing it in machines. A classical example of learning algorithms is provided by regularization in Reproducing Kernel Hilbert Spaces. The corresponding architecture however is different from the deep hierarchies found in the brain. I will sketch a new attempt (with S. Smale) to develop a mathematics for hierarchical kernel machines centered around the notion of a recursively defined derived kernel and directly suggested by the neuroscience of the visual cortex.

Relevant papers can be downloaded from http://cbcl.mit.edu/publications/index-pubs.html 